\title{
Shape Grammar Interpreter for Twisted Wire Jewelry
}

\author{
Gurpreet Kaur \\ Department of Mechanical \\ Engineering, GJ University of \\ Science and Technology \\ Hisar, India
}

\author{
Sonu Mathur \\ Department of Mechanical \\ Engineering, GJ University of \\ Science and Technology \\ Hisar, India
}

\author{
Vishal Gulati, PhD \\ Department of Mechanical \\ Engineering, GJ University of \\ Science and Technology \\ Hisar, India
}

\begin{abstract}
This work presents a Shape Grammar Interpreter to produce Indian traditional twisted wire jewelry designs implementing shape grammar approach. This formalism comprises a vocabulary of shapes and a set of shape rules that allows users to create, modify and generate twisted wire jewelry designs. The developed tool allows the designers to participate in design process through the parameter values. This framework can support the designers to create newer style of jewelry items easily and in faster way. The shape grammar approach is embedded in CAD technology to indeed help the designers in their creative jewelry designing process. This work is implemented in SOLIDWOKS using Visual Basic for Application (VBA).
\end{abstract}

\section{Keywords}

Shape Grammar, Twisted Wire Jewelry, CAD.

\section{INTRODUCTION}

Twisted wire jewelry is popular in Orissa and Andhra Pradesh in India. It is made using a technique of twisting, bending, wrapping and braiding plain precious metal wire (gold and silver) to create a lace-like effect of pierced jewelry. Standard forms of jewelry produced in this class are earrings, bracelets, brooches, pendants, chains, necklace and buttons as shown in figure 1 .
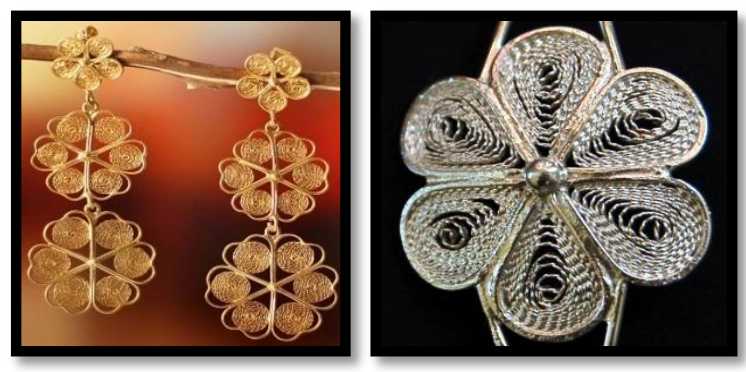

Figure 1: Twisted Wire Jewelry

This work is towards the generation of traditional class of twisted wire jewelry designs using shape grammar formalism that functions as a construction system to produce shape arrangements by employing shape rules with the aim to represent jewelry designs with variations. In fact, this work presents a Shape Grammar Interpreter (SGI) comprising a vocabulary of shapes and a set of shape rules that allows users to create, modify and generate twisted wire jewelry designs. The motivation of the work is to automate this style of jewelry for the preservation of traditional values of the craftwork. Although introducing automation into craft work will lose its charm and beauty, but it is necessary because the upcoming generations are not interested in the traditional craft work as it requires much skill and time.

\section{SHAPE GRAMMAR INTERPRETER}

Shape Grammar Interpreter represents a formalism that has captured the essence of traditional twisted wire jewelry and encoded it into a series of jewelry generations of this style. It comprises of a vocabulary of shapes and a set of shape rules analogous to traditional craftwork of twisted wire jewelry. The basic geometric shapes of motifs in traditional twisted wire jewelry are explored, developed and collected as a vocabulary (Figure 2). Further, shape rules are classified into two levels: 1) Low-level Rules and 2) High-level Rules (Figure 3). The low-level shape rules are defined as transformations like mirror, translation, copy, rotation, scaling, shear and bend (table 1). High-level rules have been developed in combination of two or more low-level rules. High-level rules represent the desired transformation as per the requirement for the generation of complex twisted wire jewelry designs. In fact graphically, high-level rules are created by concatenation of low-level transformation rules. As concatenation of transformation is non-commutative, so lowlevels rules could be applied only in a particular order to get the desired result. For example, RH is the high-level transformation created from rotation, translation, copy and bending taken in order. These rules are user defined and set of high level rules is an open ended to which new definitions can be added.

Mathematically

$$
\begin{gathered}
\mathrm{J}_{\mathrm{n}} \leftarrow \mathrm{R}_{\mathrm{H}} \mathrm{M}_{\mathrm{i}} \\
\mathrm{R}_{\mathrm{H}} \leftarrow \mathrm{R}_{\mathrm{c}} \mathrm{R}_{\mathrm{t}} \mathrm{R}_{\mathrm{c}} \mathrm{R}_{\mathrm{b}}
\end{gathered}
$$

$\mathrm{J}_{\mathrm{n}}=$ Jewelry Generations $\mathrm{Mi}=$ Shape Vocabulary $\mathrm{R}_{\mathrm{H}}=$ High Level Rule
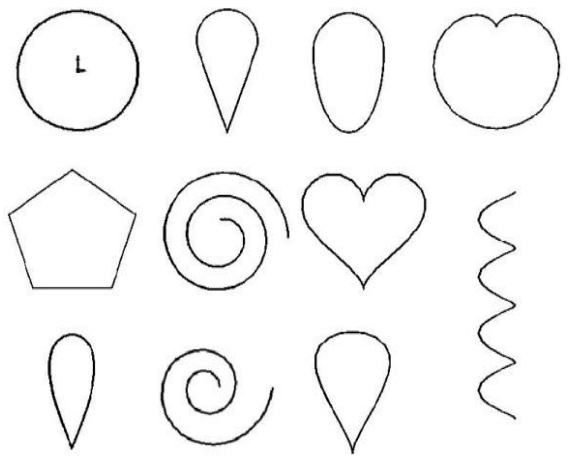

Figure 2: Vocabulary of shapes 


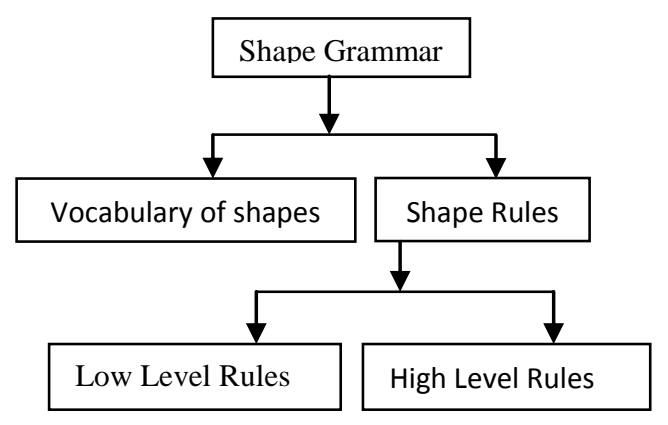

Figure 3: Shape Grammar Formalism

Table 1: Low Level Rules

\begin{tabular}{|c|l|c|}
\hline Sr. No. & Trasformations/ Rules & $\begin{array}{l}\text { Symbolic } \\
\text { Representation }\end{array}$ \\
\hline 1. & Translation & $\mathrm{R}_{\mathrm{t}}$ \\
\hline 2. & Mirror & $\mathrm{R}_{\mathrm{m}}$ \\
\hline 3. & Rotation & $\mathrm{R}_{\mathrm{r}}$ \\
\hline 4. & Scaling & $\mathrm{R}_{\mathrm{s}}$ \\
\hline 5. & Copy & $\mathrm{R}_{\mathrm{c}}$ \\
\hline 6. & Shear & $\mathrm{R}_{\mathrm{sr}}$ \\
\hline 7. & Bend & $\mathrm{R}_{\mathrm{b}}$ \\
\hline
\end{tabular}

\section{RELATED WORK}

Yufei Wang [1] presents a framework for the generation and fabrication of architectural massing designs. The framework consists of a computer program, which generates designs based on shape grammars, and rapid prototyping technologies. Lestyn Jowers [2] presented the implementation of curved shape grammars. This reflects developments of the shape grammar formalism which has traditionally been defined according to straight lines. The algorithms are implemented in a shape grammar interpreter that enables the application of shape grammars on shapes composed of quadratic Bezier curves. Seth Orsborn [3] describes how the rules for a shape grammar can be derived automatically. A statistical analysis of the design language produces fundamental shape chunks which are based on shape grammar rules. Cui Jia [4] proposes generative shape grammar approach to creating embroidery patterns. Designing process is based on a generative system with a shape grammar representation into dimensional space and representation can support human designer to create newer styles of embroidery easily, faster and visually. Trescak [5] introduced the general shape grammar interpreter for intelligent designs generations. The developed shape grammar framework allows designers to obtain automatically generated designs and to participate in the design process. Somlak Wannarumon [6] proposes a framework of generative design system for an automatic shape generation tool, which is to be used to support designers in exploring design concepts. The transformations of curved outlines are explained through shape grammar and shape rules. Xuekun Guo [7] presents an inspiration-oriented procedural approach to help artists create with abnormal structures, while preserving the semantics of biological forms.

\section{CONCEPTUALIZATION OF TWISTED WIRE JEWELRY}

The traditional piece of craftwork shown in (Figure 4) has been generated in SGI and depicted in this work as a case study. The motif in this jewelry bangle comprises of two basic geometric shapes: 1) Teardrop and 2) Spiral. The modeling parameters defining twisted wire strand, motif and jewelry type (bangle) may include size of wire, extent of twisting of wire, size of motif, size of bangle etc.

\subsection{Teardrop Geometry}

The teardrop is created by connecting a circular arc with two line segments originating from a point $\mathrm{D}$ and being tangent to the circular arc at the endpoints $\mathrm{P}_{1}$ and $\mathrm{P}_{2}$, as shown in Figure 5. The parametric form of point $\mathrm{P}_{1}$ and $\mathrm{P}_{2}$ derived as:

$$
P(x, y)=\left[r^{2} / d, \quad \frac{r}{d} \sqrt{d^{2}-r^{2}}\right]
$$

where

$\mathrm{r}=$ radius of arc,

$\mathrm{d}=$ distance between center of arc and point $\mathrm{D}$

The size of teardrop has been defined by a circumscribed rectangle of width $\mathrm{w}$ and length 1 . The parameters $\mathrm{r}$ and $\mathrm{d}$ have been conceptualized as w/2 and (1-w/2).

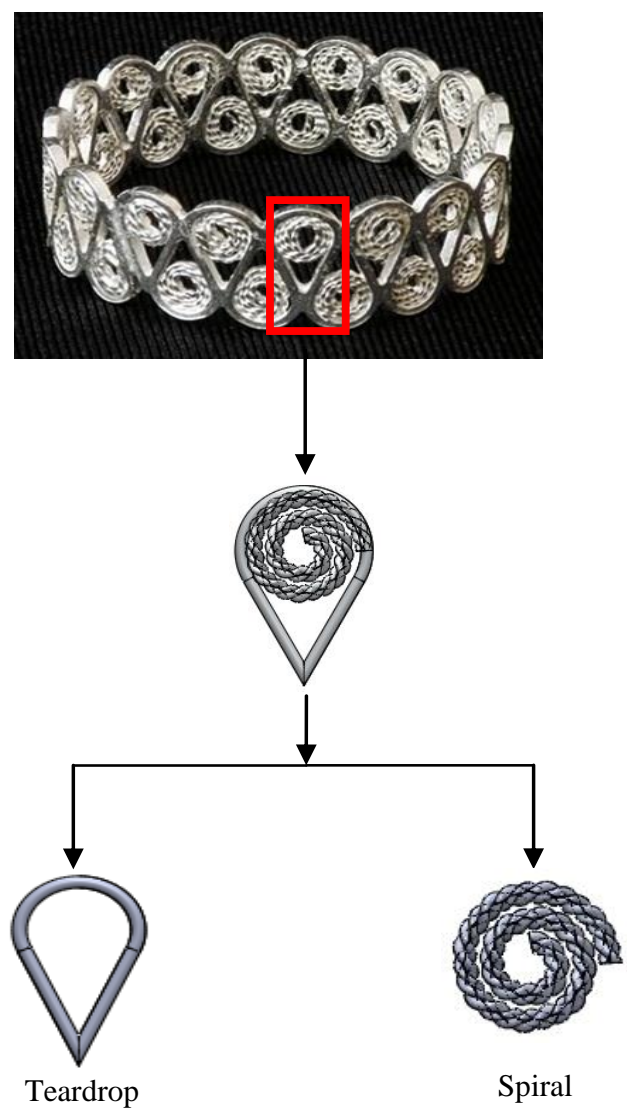

Figure 4: Traditional Piece of Twisted Wire Jewelry

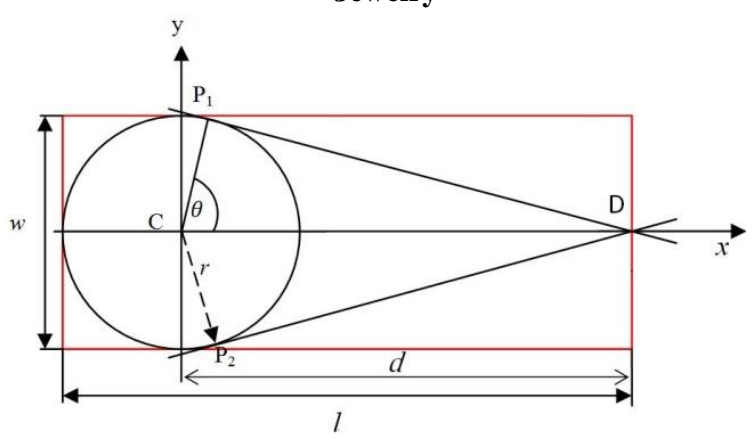

Figure 5: Teardrop Geometry 


\subsection{Spiral Geometry}

Spiral is locus of a point moving in a circle with constant speed. It starts in the origin and makes a curve with unwinded arms. The equation of the Archimedean spiral in parametric form:

$$
(x, y)=[r t \cos (2 \pi n t), r t \sin (2 \pi n t)]
$$

where

$r=$ radius of spiral,

$\mathrm{n}=$ no. of turns in spiral,

$\mathrm{t}=$ varies from 0 to 1

Affecting parameters in geometry of spiral are $r, n$ and $d$. The no. of turns $n$ are lies inside a circle of radius $r$. The distance between the two adjacent turns is equal to $\mathrm{r} / \mathrm{n}$. As twisted wire strand is constructed by sweeping a circle along the curve having diameter equal to thickness of the wire, so in order to avoid the intersection between two turns, the diameter of the wire should be less than distance $\mathrm{d}$.

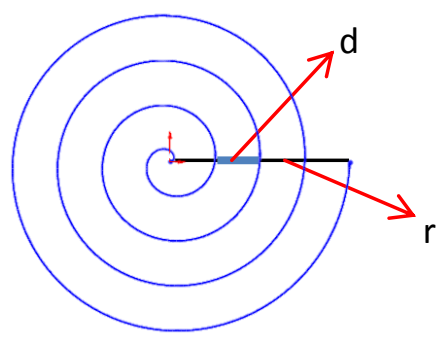

Figure 6: Spiral Geometry

\subsection{Modeling Parameters}

- Radius of wire $\left(\mathrm{R}_{\mathrm{W}}\right)$ : It is radius of wire which is mainly used to make twisted wire.

- Number of wires (c): It represents the no. of wire in twisted wire geometry.

- Number of twists in wire (T): It describes the number of twist in the wire strands of motif to form the twisted wire jewelry.

- Size of motif: It is length (l) and width (w) of rectangle in which the shapes are inscribed such as teardrop.

- Spiral size and turns: It depends upon geometry of spiral and defined as $\mathrm{r}, \mathrm{n}$ in above equation.

- Size of bangle (s): It is defined with the number of copies of motifs $(\mathrm{N})$ and equals to $\mathrm{Nw} / 2 \pi$.

- Distance $\left(\mathrm{N}_{\mathrm{d}}\right)$ : It represents the distance between numbers of copies.

\subsection{Rule Description}

$$
\begin{aligned}
& J_{n} \leftarrow R_{H} M_{i} \leftarrow\left\{R_{c} R_{t} R_{c} R_{b} M_{i}\right\} \\
& M_{i} \leftarrow\left\{S_{1}, S_{2}\right\} \\
& S_{1}=\text { Teardrop } \\
& S_{2}=\text { Sprial }
\end{aligned}
$$

\section{MODELLING APPROACH}

The modeling process includes selection of the motif shape from the library and the shape rule from the set of shape rules followed by definition of parameters through an interface created for twisted wire jewelry for the selected motif and rule. Then, modeling process starts automatically according to the modeling steps programmed to create the jewelry bangle.

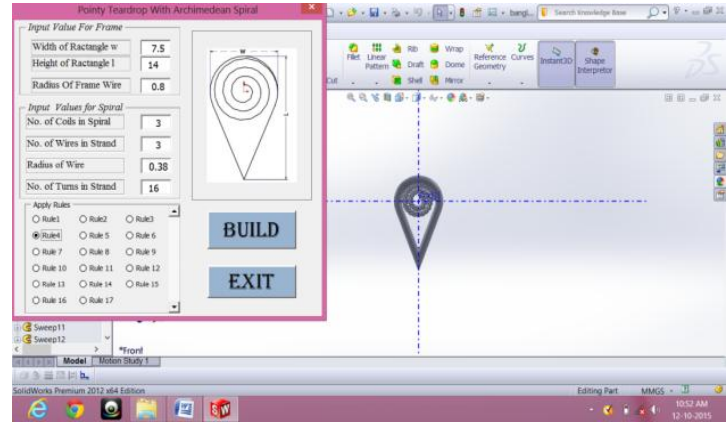

(a)

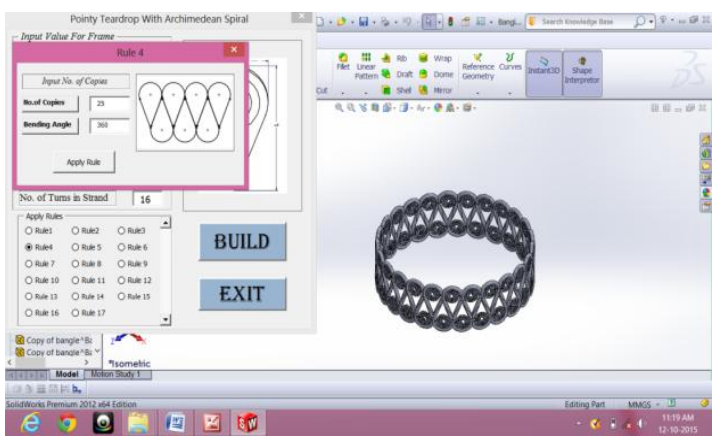

(b)

Figure 7: (a) Selection and definition of Parameters for Teardrop motif (b) Generated model of Twisted Wire Bangle

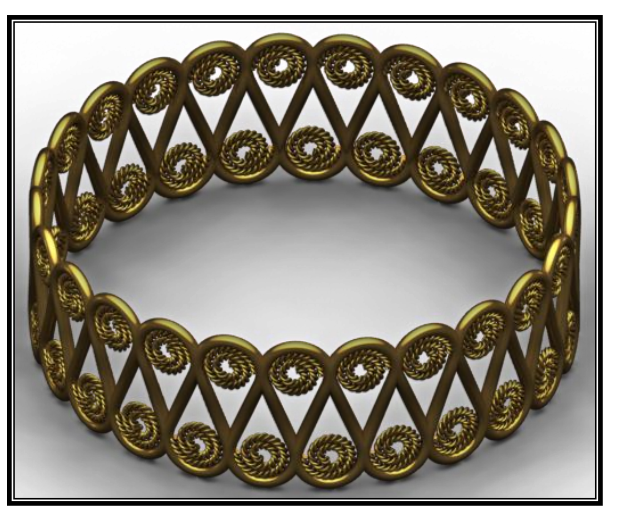

Figure 8: Rendered Image of Twisted Wire Bangle

\section{IMPLEMENTATION}

This shape grammar interpreter is implemented under the ActiveX and Visual Basic Application (VBA) programming environment using SOLIDWORKS system. The combination of the powerful ActiveX Automation object model in solidworks and VBA presents a compiling framework for customizing the software program. The design algorithm begins with mathematical description of the different geometric shapes and set of shape rules in two levels one is low level and other is high level. Then, CAD programming interfaces are used to turn the mathematical descriptions of geometric shapes and set of shape rules into computer program. These programs render the different jewelry designs on the computer screen and further the CAD data is converted into stereo lithography (STL) format, and then the STL model is ready to be submitted to a rapid prototyping machine for manufacturing. Rapid prototyping model of twisted wire bangle is shown in the (Figure 9). 


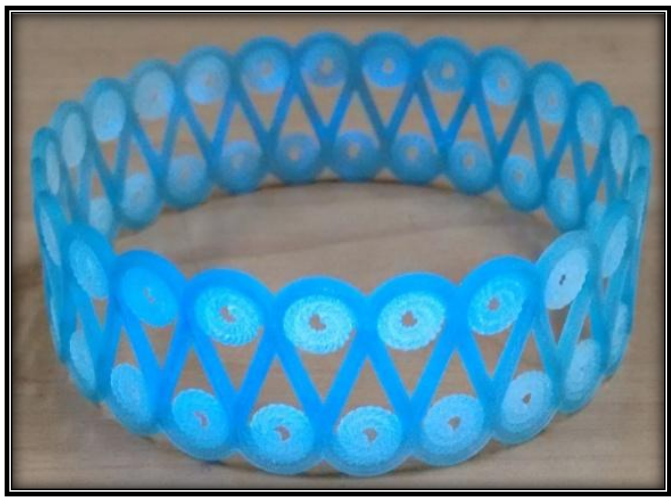

Figure 9: Rapid Prototyping Model of Twisted Wire Bangle

\section{CONCLUDING REMARKS}

This work has created a generative production system using shape grammar methodology for emergent jewelry shapes to support the designers in a creative and intuitive manner. By extracting appropriate features (parameters), a large number of design solutions with new styles have been generated. To enhance the communication between designer and Interpreter, a three-dimensional evolutionary modeling approach has been developed for the exploration of new designs. The CAD technology, embedding shape grammar as the formal approach, would indeed help the designers in their creative design process. The future work can be extended to parameterize more of the different type of jewelry designs generally used in jewelry sector.

\section{REFERENCES}

[1] Yufei Wang and Jose Pinto Duarte, Automatic generation and fabrication of designs, Automation in construction, Vol. 11, pages 291-302, 2002.

[2] Lestyn Jowers and Christopher Earl, Implementation of curved shape grammars, Environment and Planning and Design, 38(4) pp, 616-635.

[3] Seth Orsborn, Jonathan Cagan and Peter Boatwright, Automating the creation of shape grammar rules, Design Computing and Cognition, 2008.

[4] Cui Jia and Tang Ming XI, Chinese Pattern Design Using Generative Shape Grammar, 13th Generative Conference GA 2010.
[5] T.Trescak, I.Rodriguez and M.Esteva, General shape grammar interpreter for intelligent designs generations, Artificial Intelligence Research Institute, Barcelona, Spain, 2011.

[6] Somlak Wannarumon, Prapasson Pradujphongphet and Erik J. Bohej, The framework of generative system using shape grammar for jewelry design, International journal of intelligent information processing, vol.4, no.2, June 2013.

[7] Xuekun Guo, Juncong Lin, Kai Xu and Xiaogang Jin, Creature grammar for creative modeling of 3D monsters, Graphical Models, Vol. 76, pages 376-389, 2014.

[8] Filipe Santos and Joaquim Esmerado, A Different Shape Grammar Approach for Automatic Design Generation, International Journal of Advances in Computer Science \& Its Applications, Volume 5: Issue 1, April, 2015.

[9] Dhuha A, Al-kazzaz and Alan H. Bridges, A framework for adaptation in shape grammars, Design Studies, vol.33, pages 342-356, 2012.

[10] Sehnaz Cenani and Gulen Cagdas, Shape grammar of geometric Islamic ornaments, Architectural Design Computing Graduate Program, Turkey, session 7, 2009.

[11] V.Gulati, P.Tandon, and H. Singh, A parametric voxel based unified modeler for creating carved jewelry, Computer Aided Design and Applications; 5(6), 811821, 2008.

[12] V.Gulati, P.Tandon and H. Singh, A Jewelry Modeler for the Fret-worked Bangles, International Journal of Computer Applications, 2(2), pages 76-80, 2010.

[13] G.Stiny and J.Gips, Shape grammar and generative specifications of painting and sculpture, In C.V. Friedman, editor, Information Processing vol. 71, pages 1460-1465, Amsterdam, 1972.

[14] Agarwal and Cagan, A blend of different tastes: The language of coffee makers, Environment and Planning B: planning and design, Vol.25, no. 2, pages 205-226, 1998.

[15] M. Smytha and E. Edmondsb, Supporting design through the strategic use of shape grammars, Knowledge-Based Systems, Vol.13, pages 385-393, 2000. 